\title{
Subserous invasion of VEGF-C-producing cancer cells is a possible risk factor for ileal ulceration in the non-metastatic mucosal layer during bevacizumab-combined chemotherapy for recurrent ovarian cancer: A case report
}

\author{
TAKEO MATSUMOTO, YASUNARI MIZUMOTO, KYOHEI NAKADE, TAKESHI OBATA, AYUMI MATSUOKA, \\ SUBARU MYOJO, MASANORI ONO, MITSUHIRO NAKAMURA and HIROSHI FUJIWARA
}

Department of Obstetrics and Gynecology, Kanazawa University Graduate School

of Medical Science, Kanazawa, Ishikawa 920-8641, Japan

Received May 3, 2017; Accepted July 13, 2017

DOI: $10.3892 /$ mco.2017.1403

\begin{abstract}
A 65-year-old woman received chemotherapy using taxane and carboplatin prior and following optimal debulking surgery for ovarian cancer stage IV. Five months later, intra-abdominal recurrence was diagnosed, and second-line chemotherapy using nogitecan and bevacizumab was administered. After five courses, the patient presented with a symptom of subileus and subsequent intestinal perforation occurred. An emergent surgery revealed two perforation sites and longitudinally extended ulcerative lesions in the ileum. Pathologically, although metastatic sites were not observed in the submucus layer just beneath the ulcers, there were a number of vascular endothelial growth factor (VEGF)-C-positive cancer cell invasion sites along with marked edema and an increase of the lymphatic endothelial cell marker 'podoplanin'-positive cells in subserous regions. Since bevacizumab is able to inhibit VEGF-A, but not VEGF-C, and induce compensatory increase in VEGF-C production, these findings suggest that the local disturbance of lymphatic circulation in the subserous regions by VEGF-C-producing cancer cells is a possible risk factor for the development of intestinal ulceration and perforation during bevacizumab therapy.
\end{abstract}

\section{Introduction}

Bevacizumab, an anti-vascular endothelial growth factor-A (VEGF-A) antibody, is one of the most commonly available

Correspondence to: Dr Yasunari Mizumoto, Department of Obstetrics and Gynecology, Kanazawa University Graduate School of Medical Science, 13-1 Takaramachi, Kanazawa, Ishikawa 920-8641, Japan

E-mail: yas1025@med.kanazawa-u.ac.jp

Key words: bevacizumab, gastrointestinal ulcer, ovarian cancer, perforation, subserous invasion, VEGF-C reagents for molecular-targeted therapy (1). It was reported that the therapeutic mechanisms of bevacizumab are fundamentally based on its inhibitory effects on neovascularization. In several fields, this drug is used for less-invasive therapy against malignant diseases, expecting to reduce the blood supply of cancer tissues without reducing the drug delivery efficiency of the concurrently administered anticancer drugs. Recently, bevacizumab was also applied for platinum-resistant recurrent ovarian cancers in combination with second-line anticancer drugs. According to the AURELIA (Avastin Use in Platinum-resistant Epithelial Ovarian Cancer) open-label randomized phase III trial, progression-free survival was extended 2-fold compared with anticancer drug use alone $(2,3)$.

Although bevacizumab does not induce severe acute side effects (4), its usage with anticancer drugs is associated with non-negligible pathological conditions such as gastrointestinal perforation (5). Recently, we encountered a case of intestinal perforation at the ileum after the fifth course of second-line chemotherapy using nogitecan and bevacizumab for platinum-resistant recurrent ovarian cancer $(2,3)$. The perforated sites were surrounded by broad and longitudinal ulcer lesions without apparent features showing direct invasion or apoptosis of cancer cells beneath the mucosal layer. Microscopic examination of the lesion sites around perforation revealed diffuse invasion of VEGF-C-positive cancer cells in the subserous regions along with marked edema and an increase of podoplanin-positive cells. Since bevacizumab can inhibit VEGF-A, but not VEGF-C, and could induce compensatory increase in VEGF-C production (6), the findings suggested that the subserous involvement of VEGF-C-producing ovarian cancer cells is a new risk factor for the development of intestinal ulceration and subsequent perforation during combined therapy with an anticancer drug and bevacizumab. Consequently, we provide precise clinical and pathological information concerning this case.

\section{Materials and methods}

Tissue samples and patients. The surgical specimens were fixed with $20 \%$ formalin and embedded in paraffin. The tissue 
sections $(4 \mu \mathrm{m})$ were stained by routine histopathological techniques for diagnosis. Representative tissue sections of each specimen were subjected to immunohistochemical examination in this study. Informed consent for the use of these tissues in this study was obtained from the patient.

Immunohistochemistry. Tissue localization of VEGF-C, podoplanin, and CD34 was immunohistochemically determined by the avidin-biotin-peroxidase complex (VECTASTAIN ABC Kit, Vector Laboratories, Burlingame, CA, USA) method according to the manufacturer's protocol. Briefly, the sections of representative blocks were deparaffinized in xylene, rehydrated in ethanol, and antigen retrieval was subsequently performed in $0.01 \mathrm{M}$ citrate buffer, $\mathrm{pH}$ 6.0. The slides were immersed in $3 \%$ hydrogen peroxide for $10 \mathrm{~min}$ to block endogenous peroxidase activity and then washed in $0.05 \mathrm{~mol} / \mathrm{l}$ phosphate-buffered saline (PBS), $\mathrm{pH}$ 7.4. The slides were incubated with primary antibody, anti-human VEGF-C polyclonal goat antibody (R\&D Systems, MN, USA) at a concentration of $5 \mu \mathrm{g} / \mathrm{ml}$, anti-human podoplanin monoclonal mouse antibody, clone D2-40 (Dako, Tokyo, Japan) at a concentration of $5 \mu \mathrm{g} / \mathrm{ml}$, or anti-human CD34 monoclonal mouse antibody, class II (Dako, Tokyo, Japan) at a concentration of $5 \mu \mathrm{g} / \mathrm{ml}$ overnight at $4^{\circ} \mathrm{C}$ in a humidified chamber, respectively. Control staining was obtained by replacing the primary antibody by normal serum solution. After washing, the sections were incubated for $30 \mathrm{~min}$ with biotin-labeled rabbit anti-goat IgG for VEGF-C or horse anti-mouse IgG for podoplanin and CD34 at room temperature, respectively. Then, they were treated with the avidin-biotin complex at room temperature. Sites of peroxidase activity were visualized with diaminobenzidine (Liquid DAB+Substrate Chromogen System, Dako, Carpinteria, CA, USA), and the sections were counterstained with hematoxylin.

\section{Case report}

A 65-year-old woman (2 gravida, 2 para) visited Kanazawa University Hospital with a clinical diagnosis of ovarian cancer. After 3 cycles of neoadjuvant chemotherapy involving DC therapy (docetaxel and carboplatin), the patient underwent hysterectomy with bilateral salpingo-oophorectomy, omentectomy, appendectomy, and reduction surgery of the peritoneally implanted cancer masses. The pathological diagnosis was serous adenocarcinoma. Six additional courses of chemotherapy with TC therapy (paclitaxel and carboplatin) were administered. During the clinical course, serum CA125 was reduced from 12,770 to $<35 \mathrm{IU} / \mathrm{ml}$. However, 5 months later, intra-abdominal recurrence occurred with the elevation of serum CA125 to 1,334 IU/ml. Accordingly, second-line chemotherapy using nogitecan was performed in combination with bevacizumab. Although serum CA125 was successfully reduced within 2 courses, its serum value was maintained at approximately $100 \mathrm{IU} / \mathrm{ml}$ without being reduced to the normal range during this sequential regimen.

After the fifth course, the patient presented with subileus and a mild fever. Several peritoneal recurrent masses were detected by computed tomography (CT). The administration of bevacizumab was immediately discontinued and supportive care, including administration of antibiotics, was started. However, 17 days later, intestinal perforation occurred with acute abdom- inal pain. An emergent operation demonstrated the presence of two nearby bowel perforations in the ileum concomitant with diffuse peritoneal recurrent lesions in the abdominal cavity. At the distal site of perforations in the ileum, severe stenosis due to peritoneal adhesion by cancer dissemination was observed. Partial small bowel resection and ileostomy were performed. In the resected ileum, longitudinal and segmental ulcerative lesions were observed in the luminal face, and two perforation sites were located in the distal area of the ulcerative lesions (Fig. 1A). Later, pathological examination revealed that there were a number of cancer cell invasions in the subserous regions, which were located on the reverse side of the ulcerative lesions at the inner lumen site (Fig. 1B-D). Although there were marked numbers of metastatic foci in the intramesenteric regions, microscopic examinations could hardly detect the metastatic lesions on the serous surface in the resected ileum. On the other hand, cancer foci were abundant in the subserous regions (Fig. 1B-D). Notably, the expression of immunoreactive VEGF-C was observed on the invading cancer cells in the subserous regions (Fig. 2A-B). In accordance with these findings, marked edematous changes and an increase of podoplanin-positive cells were frequently observed in the subserous areas between the serous surface and muscle layer (Fig. 2C-D). However, the post-operative control of intra-abdominal infection and intestinal ulceration was difficult, and the patient died 2 months after the perforation.

\section{Discussion}

Gastrointestinal perforation is one of the most severe complications of bevacizumab. Accordingly, in cases of ovarian primary cancer, it has been reported that bowel obstruction symptoms, rectosigmoid involvement, bowel involvement, and wall thickening on $\mathrm{CT}$, pretreatment with 3 or more regimens, and platinum-resistant disease are risk factors for intestinal perforation. It is also well-known that intestinal edematous changes are frequently observed in cases with diarrhea and mechanical obstruction. However, it is occasionally difficult to predict the potential risk accurately and prevent the onset of perforation. Indeed, in this case, although the administration of bevacizumab was immediately discontinued after detecting the subileus symptoms, the supportive care could not avoid bowel perforation and the subsequent peritoneal leakage from the small intestine.

Notably, antibiotic is also a reported risk factor for bowel perforation in the patient heavily treated with bevacizumab by unknown mechanisms (7). In the present case, although we have treated patient with antibiotics for subileus symptom with mild-fever and elevated WBC counts, the bevacizumab was only administrated for 5 courses, that the contribution of antibiotics to intestinal perforation is unclear.

Macroscopic and microscopic pathological examinations of the resected intestine revealed the marked subserous involvement of ovarian cancer cells. The metastatic masses that directly invaded from the serosa to mucosa were not observed around the two perforated sites. In addition, although there were longitudinal and segmental ulcerations, we could hardly observe cancer cell invasion in the mucosal layer. Multiple ulcerations and bowel perforation by bevacizumab were reported to develope during chemotherapy for breast and lung cancers even under cancer-free conditions 

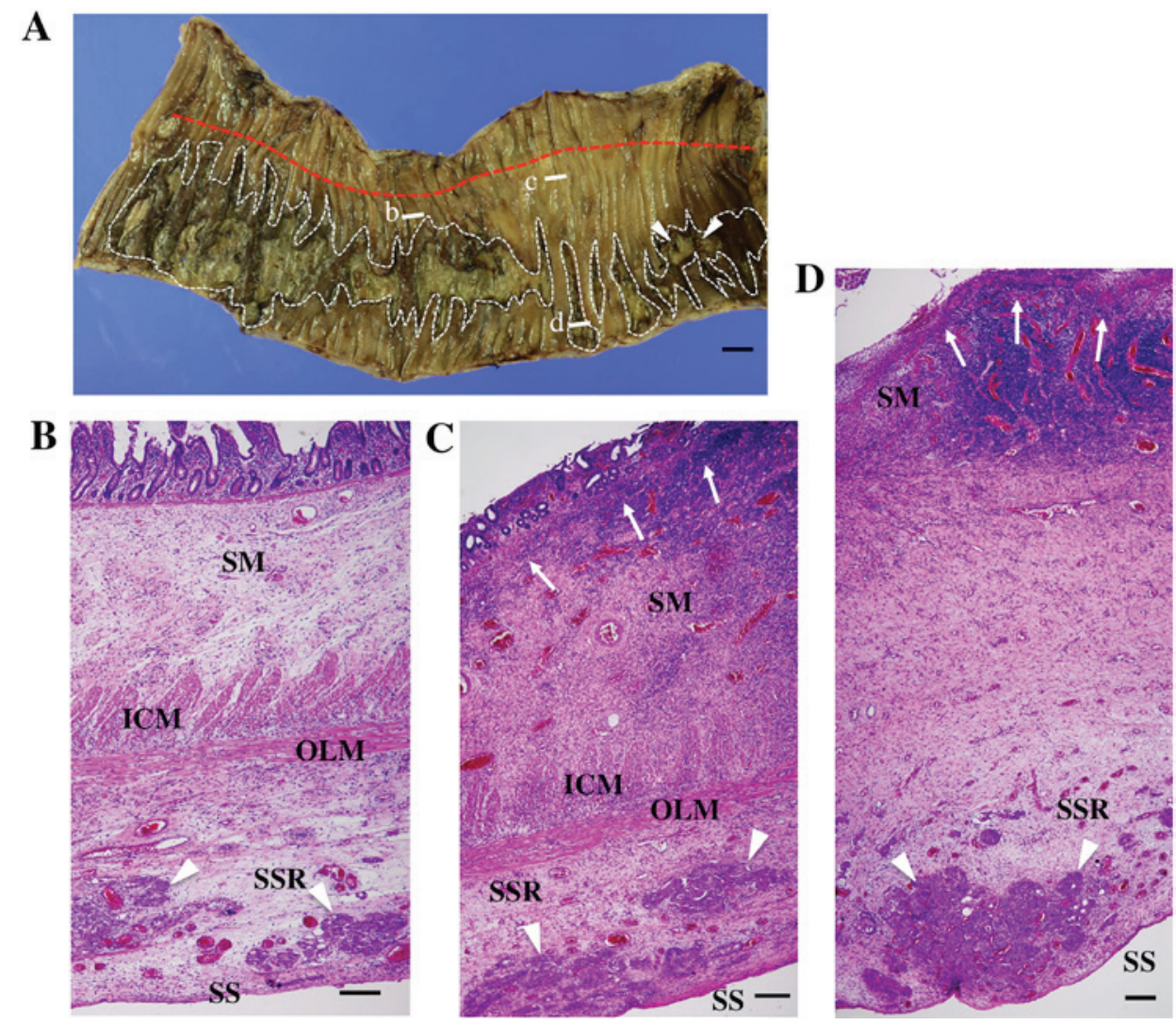

Figure 1. Macroscopic and microscopic images of the resected ileum. (A) a macroscopic image of the resected ileum from the luminal aspect. Longitudinal and segmental ulcerative lesions were observed in the luminal face (white dotted lines). Two sites of perforation were detected in the distal region (white arrowheads). The attachment line of the mesenterium at the opposite site is shown by a red dotted line. White bars b, $\mathrm{c}$, and $\mathrm{d}$ show the sites of the following microscopic images of B-D, respectively. (B-D) microscopic images of the resected ileum. (B) a non-ulcerative lesion. Cancer cell invasion (white arrowheads) with stromal edema was observed in the subserous region (SSR). Although the submucous region (SM) was also edematous, the structures of the mucosa, mucosal muscle, inner circular (ICM), and outer longitudinal muscle layer (OLM) were maintained. C, an erosive lesion progressing toward ulceration. Damage of the mucosal muscles was observed (white arrows). (D) an ulcerative lesion. The mucosal muscles were directly exposed to the luminal cavity (white arrows), and the structures of the inner circular and outer longitudinal muscle layer became obscure. On the other hand, the subserous lesion showed marked edema with the cancer cell masses (white arrowheads). SM, submucous region; ICM, inner circular muscle layer; OLM, outer longitudinal muscle layer; SSR, subserous region; SS, serous surface. Black bars show $1 \mathrm{~cm}$ (A) and $200 \mu \mathrm{m}$ (B-D), respectively.

in the abdomen. Accordingly, it should be noted that the combination therapy of an anticancer drug with bevacizumab adversely works to maintain the physiological homeostasis of the intestinal mucosal layer, where certain additional exacerbating factors can induce mucosal disorders such as ulcerations and perforation.

In the present case, severe edema was widely observed in the subserous regions along with invading cancer cell foci in the resected ileum, suggesting poor lymphatic return and drainage around these areas. In general, since the epithelium of the small intestinal has high proliferative properties, the direct attack of epithelial cells by anticancer drugs frequently causes mucosal ulceration. Under these conditions, the inhibitory effects of bevacizumab on neovascularization can exacerbate the ulceration by interfering with the repair process. In addition to the above mechanisms, it should be noted that lymphatic dysfunction was reported to affect ulcerative disorders such as Crohn's disease. Anatomically, the small intestine has two distinct lymphatic networks: A submucosal network including the lacteals, and that of the muscle layer (8). Although these two lymphatic networks have no direct connections, both systems drain into common collecting lymphatics in the subserous regions near the mesenteric border of the intestine (9). It was reported that lymphangitis was one of the earliest pathological findings in Crohn's disease (10), and that the sclerosis of regional intestinal lymphatics can induce the onset of Crohn's disease in pigs (11). Lymphangiogenesis and lymphangiectasia were frequently associated with ileal and colonic Crohn's disease (12). Consequently, it is theoretically possible that edematous changes caused by poor lymphatic return and drainage in the subserous regions may subsequently lead to a disturbance of the distant lymphatic microenvironment of the mucosal layer, causing chronic ulcerative lesions.

When the intestine has chronic ulcerative lesions, it is reasonable to speculate that a continuous mechanical stress at the proximal site of the narrowing region would trigger intestinal perforation. This mechanical stress may also contribute to further edematous changes in the ileum. However, considering the coexistence of edematous changes with cancer cell invasion in the subserous regions, it is more appropriate to suggest that cancer cell invasion would be mainly responsible for the subserous edema. In this case, the metastatic pathway along the subserous regions remains unknown. Although a large number of cancer cell foci in the intramesenteric regions can be a candidate for a local origin, 
A

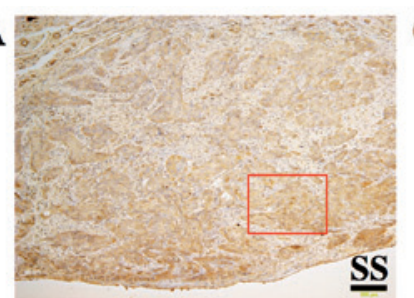

B

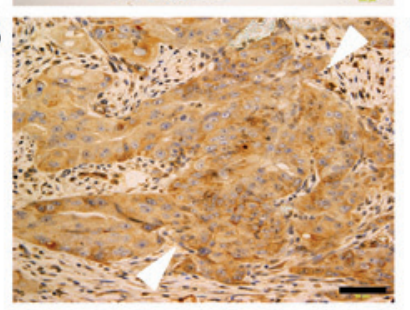

I

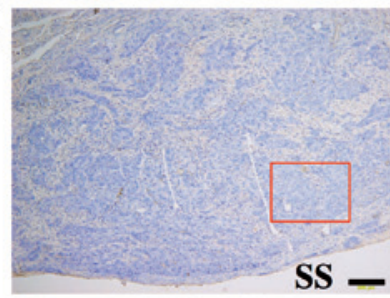

J

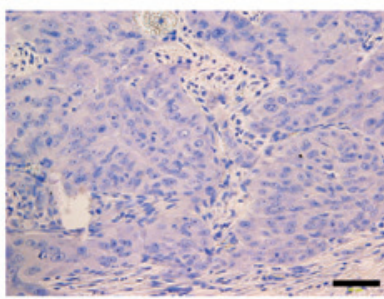

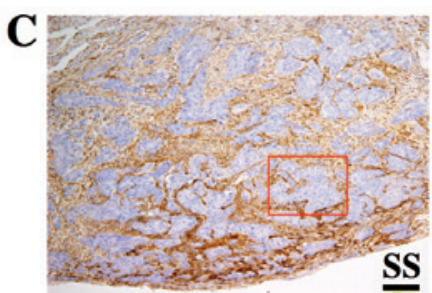

\section{D}

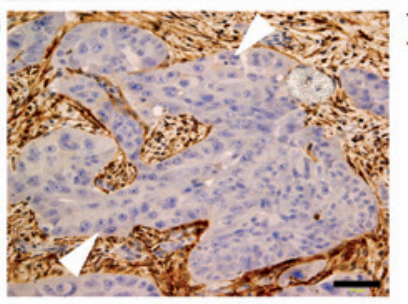

E

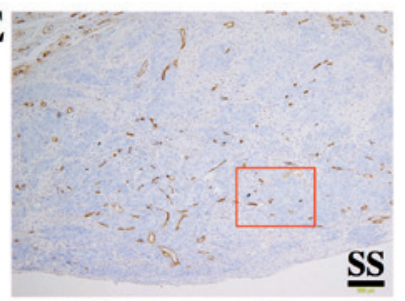

F

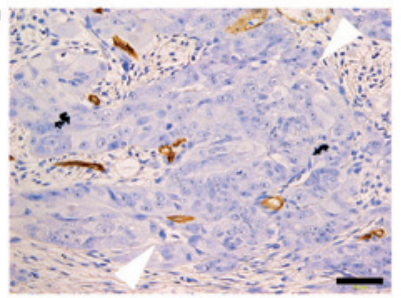

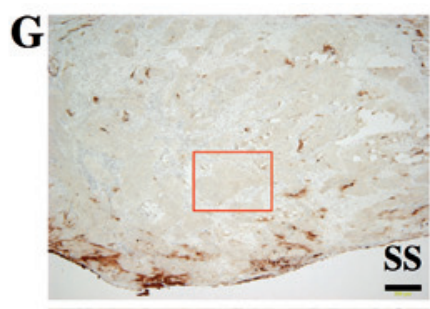

H

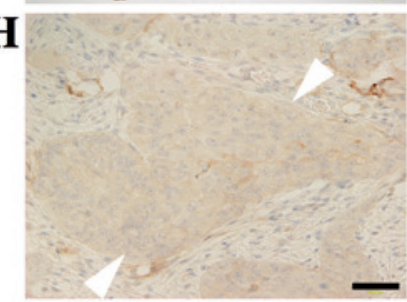

K

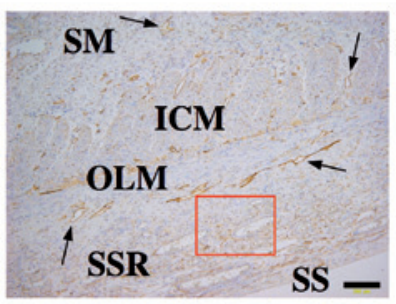

L

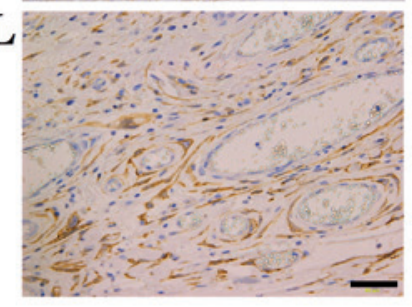

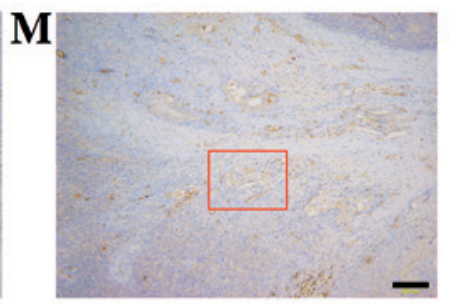

$\mathbf{N}$

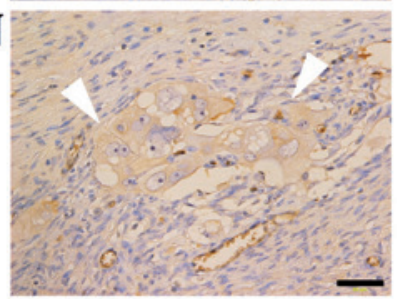

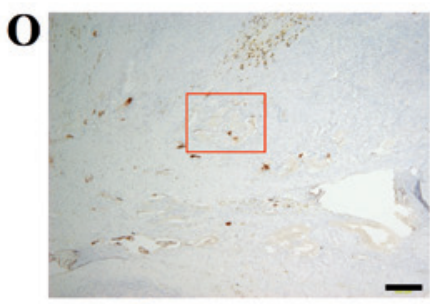

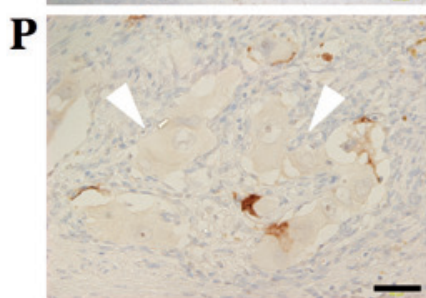

Figure 2. Immunoreactive expression of VEGF-C, podoplanin, and CD34 in the resected ileum. (A-J) a metastatic lesion in the subserous area. (B, D, F, $\mathrm{H}$ and $\mathrm{J}$ ) are magnified images of the areas within the red squares of (A, C, E, G and I, respectively). (A and B) immunoreactive expression of VEGF-C was observed on the invaded cancer cells (white arrowheads). (C and D) an increase of podoplanin-positive cells was detected around invading cancer cells (white arrowheads). (E and F) CD34-positive vascular endothelial cells were recognized among invaded cancer cells (white arrowheads). (G and H) weak VEGF-A expression were observed in metastatic region. (I and J) negative controls. (K and L) a non-metastatic lesion. (K) podoplanin-positive lymphatic vessels were clearly observed in the submucous region, inner circular muscle layer, and outer longitudinal muscle layer (black arrows). (L) Although apparent cancer invasion was not detected, podoplanin-positive fibroblast-like cells were observed in the subserous region. (M and N) Primary ovarian lesion at the initial operation after NAC therapy. (O and P) positive staining of VEGF-A were also observed in primary tumor. The immunoreactive expression of VEGF-C was observed on the surviving cancer cells (white arrowheads). SM, submucous region; ICM, inner circular muscle layer; OLM, outer longitudinal muscle layer; SSR, subserous region; SS, serous surface. Black bars show $200 \mu \mathrm{m}$ (A, C, E, G, I, and K) and $50 \mu \mathrm{m}(\mathrm{B}, \mathrm{D}, \mathrm{F}, \mathrm{H}, \mathrm{J}$, and L), respectively.

this study provides no evidence for retrograde lymphatic progression toward the subserous from intramesenteric regions.

It is well-known that VEGF families also play important roles in lymphangiogenesis. The activation of VEGF receptor-2 (VEGFR-2) and VEGFR-3 by VEGF-C and -D was reported to initiate lymphangiogenesis by promoting lymphatic proliferation and migration and by organizing new lymphatic endothelial cells into functional capillaries Accordingly, the coneutralization of VEGFR-2 and VEGFR-3 completely inhibited lymphatic organization (13). Importantly, although bevacizumab is an anti-VEGF-A antibody, this agent was demonstrated to induce the compensatory expression of VEGF-C and -D in glioma cells, suggesting that VEGF-C and -D facilitate escape from bevacizumab therapy (6). In this case, immunoreactive VEGF-C, but not VEGF-D (data not shown), was observed in the invading cancer cells. The intensity of VEGF-C expression in the subserous metastatic region was considered to be higher than that of the primary lesion (Fig. 2K and L). VEGF-D expression were negative both in primary and metastatic regions (data not shown). In addition, the abundant proliferation of spindle shape cells that were positive for a lymphatic endothelial cell marker, podoplanin, was also observed in the metastatic regions. Therefore, it can be speculated that bevacizumab directly interferes with the angiogenic environment around the affected lesions, and then induces compensatory production of VEGF-C by cancer cells, leading to a local disturbance of the lymphatic environment by poorly controlled lymphoangiogenesis.

In conclusion, this case suggests that local disturbance of the lymphatic function in subserous regions associated with cancer cell invasion can be an additional risk factor to promote the intestinal ulceration and perforation during bevacizumab therapy. Clinical signs of risk factors for gastrointestinal perforation, such as subileus symptom and thickness of the bowel wall, may represent progressive functional disorders of intestinal muscles and edematous change derived from a poor lymphatic return and drainage. This study also demon- 
strates the possible involvement of VEGF-C-producing cancer cells in the edematous changes. Further investigation of mechanisms leading to the local disturbance of the lymphatic function in subserous regions may contribute to developing new approaches to predict or avoid gastrointestinal perforation during bevacizumab therapy.

\section{Acknowledgements}

The present study was supported in part by Grants-in-Aid for Scientific Research (no. 26293358). The authors appreciate Associate Professor Hiroko Ikeda at the Division of Pathology, Kanazawa University Hospital for her valuable advice.

\section{References}

1. Reinthaller A: Antiangiogenic therapies in ovarian cancer. Memo 9: 139-143, 2016

2. Pujade-Lauraine E, Hilpert F, Weber B, Reuss A, Poveda A, Kristensen G, Sorio R, Vergote I, Witteveen P, Bamias A et al: Bevacizumab combined with chemotherapy for platinum-resistant recurrent ovarian cancer: The AURELIA open-label randomized phase III trial. J Clin Oncol 32: 1302-1308, 2014.

3. Husain A, Wang Y, Hanker LC, Ojeda B, Anttila M, Breda E Vuylsteke P and Pujade-Lauraine E: Independent radiologic review of AURELIA, a phase 3 trial of bevacizumab plus chemotherapy for platinum-resistant recurrent ovarian cancer. Gynecol Oncol 142: 465-470, 2016.

4. Martin JY, Urban RR, Liao JB and Goff BA: Bevacizumab toxicity in heavily pretreated recurrent epithelial ovarian, fallopian tube, and primary peritoneal cancers. J Gynecol Oncol 27: e47, 2016.
5. Keating GM: Bevacizumab: A review of its use in advanced cancer. Drugs 74: 1891-1925, 2014.

6. Grau S, Thorsteinsdottir J, von Baumgarten L, Winkler F, Tonn JC and Schichor C: Bevacizumab can induce reactivity to VEGF-C and -D in human brain and tumour derived endothelial cells. J Neurooncol 104: 103-112, 2011.

7. Ogata K, Takamori H, Umezaki N, Yagi T, Ogawa K, Ozaki N, Hayashi H, Tanaka H, Ikuta Y, Doi K: Gastrointestinal perforation during regorafenib administration in a case with hepatic metastases of colon cancer. J Chemother 20:1-3, 2016

8. Miller MJ, McDole JR and Newberry RD: Microanatomy of the intestinal lymphatic system. Ann N Y Acad Sci 1207 (Suppl 1): E21-E28, 2010.

9. Unthank JL and Bohlen HG: Lymphatic pathways and role of valves in lymph propulsion from small intestine. Am J Physiol 254: G389-G398, 1988.

10. von der Weid PY, Rehal S and Ferraz JG: Role of the lymphatic system in the pathogenesis of Crohn's disease. Curr Opin Gastroenterol 27: 335-341, 2011.

11. Kalima TV, Saloniemi H and Rahko T: Experimental regional enteritis in pigs. Scand J Gastroenterol 11: 353-362, 1976.

12. Pedica F, Ligorio C, Tonelli P, Bartolini S and Baccarini P: Lymphangiogenesis in Crohn's disease: An immunohistochemical study using monoclonal antibody D2-40. Virchows Arch 452: 57-63, 2008.

13. Holzheimer RG and Mannick JA (eds): Surgical Treatment: Evidence-Based and Problem-Oriented. Part II: Small bowel obstruction. W. Zuckschwerdt Verlag GmbH, Munich, 2001.

14. Goldman J, Rutkowski JM, Shields JD, Pasquier MC, Cui Y, Schmökel HG, Willey S, Hicklin DJ, Pytowski B and Swartz MA: Cooperative and redundant roles of VEGFR-2 and VEGFR-3 signaling in adult lymphangiogenesis. FASEB J 21: 1003-1012, 2007. 